\title{
People with Parkinson's Disease: What Symptoms Do They Most Want to Improve and How Does This Change with Disease Duration?
}

\author{
Rebecca J. Port ${ }^{\mathrm{a}}$, Martin Rumsby ${ }^{\mathrm{b}}$, Graham Brown $^{\mathrm{b}}$, Ian F. Harrison ${ }^{\mathrm{c}}$, Anneesa Amjad $^{\mathrm{a}}$ \\ and Claire J. Bale ${ }^{\mathrm{a}, *}$ \\ ${ }^{\text {a }}$ Parkinson's UK, London, UK \\ ${ }^{\mathrm{b}}$ Research Support Network, London, UK \\ ${ }^{\mathrm{c}}$ Centre for Advanced Biomedical Imaging, Department of Imaging, Division of Medicine, University College \\ London, London, $U K$
}

Accepted 16 December 2020

Pre-press 11 January 2021

\begin{abstract}
.
Background: Parkinson's disease (PD) is a neurodegenerative condition with a diverse and complex pattern of motor and non-motor symptoms which change over time with disease duration.

Objective: The aims of the present study were to discover what symptoms matter most to people with the condition and to examine how these priorities change with disease duration.

Methods: A simple free-text online survey (using SmartSurvey) was developed by Parkinson's UK, which asked participants to identify up to three aspects of the condition they would most like to see improvement in.

Results: 790 people participated reporting 2,295 issues related to PD which were grouped into 24 broad symptom domains. Of these, 1,358 (59.1\%) were categorised as motor symptoms, 859 (37.4\%) as non-motor issues and $78(3.4 \%)$ as medication problems. This study reveals how certain features of PD become more or less important to patients as the condition progresses. Non-motor symptoms were highly cited from the very earliest stages of PD. Problems with walking, balance and falls, speech problems, freezing and dyskinesia become increasingly important as the condition progresses whereas tremor, stiffness and psychological health become decreasingly important as the condition progresses.

Conclusion: The data suggest that the priorities of people affected by PD for improving life are personal and change with duration of the condition. These findings have implications for developing person-centred management and care, as well as for directing future research to improve quality of life.
\end{abstract}

Keywords: Parkinson's disease, quality of life, symptoms, patient priorities, progression

\section{INTRODUCTION}

Parkinson's disease (PD) is an extremely complex,

*Correspondence to: Claire Bale, Parkinson's UK, 215 Vauxhall Bridge Road, London SW1V 1EJ, UK. Tel.: +44 207963 9326; E-mail: cbale@parkinsons.org.uk. progressive neurodegenerative condition that, in the UK, affects about 145,000 people with a male to female ratio of about $3: 2$ [1]. PD is characterised by a 
broad range of motor and non-motor symptoms [2-4]. Clinical observations suggest that two major subtypes of PD can be defined, namely tremor-dominant PD with a relative absence of other motor symptoms and non-tremor dominant PD. A subgroup of PD patients has an intermediate phenotype with several motor symptoms [3]. As the condition progresses, the number and severity of symptoms increases. The amount of medication required to manage symptoms also increases leading to a risk of side effects and fluctuations contributing to increased disability [4-7].

While PD has some common features-namely the cardinal motor symptoms of tremor, rigidity and bradykinesia-it is clinically highly heterogeneous, with each individual experiencing their own unique blend of symptoms and side effects [8]. Large-scale cohort studies are underway that aim to track and record the evolution of PD over time to try and understand this heterogeneity, and identify subtypes of the condition based upon the symptoms experienced and how these symptoms evolve over time [9]. However, alongside these studies, it is essential to understand the symptoms and complications of the condition that are most troublesome and distressing to patients and those close to them, rather than simply focusing on those that are most common.

Previous research undertaken in this area has sought to identify research priorities for PD, including the James Lind Alliance priority setting partnership carried out by Parkinson's UK in 2014 [10]. This study revealed that the top ten research priorities for PD management included the need to address motor symptoms (balance and falls, and fine motor control), non-motor symptoms (sleep and urinary dysfunction), mental health issues (stress and anxiety, dementia and mild cognitive impairments), side effects of medications (dyskinesia) and the need to develop interventions specific to the phenotypes of PD and better monitoring methods.

Such studies have identified key areas of unmet need for the PD population but do not give us a picture of how patient priorities evolve as the condition progresses. Our present study was undertaken to examine this particular problem in more depth.

\section{MATERIALS AND METHODS}

Study design

Participants were people with PD, partners, carers or family members answering about a person with PD. The data were collected from 11/04/2018 to $02 / 05 / 2018$ using an online survey.

The study was advertised using the Parkinson's UK Research Support Network, with an email list of around 4,800 members with a connection to PD at the time of survey advertisement. The network is primarily UK based but no exclusion was placed on location. The only inclusion criteria were that the individual should be able to read and write in English.

\section{Ethics}

No ethical consent was required to carry out this study as the data were submitted anonymously and all survey respondents agreed to a disclosure statement. Members of the Parkinson's UK Research Support Network had given prior consent to receive correspondence from the charity.

\section{Content of the survey}

The aim was to produce a survey that could be quickly and easily completed to achieve the largest possible response. The final survey was designed to be completed in under 5 minutes. Respondents were required to read and agree to a survey purpose and disclosure statement. Those who chose to proceed were presented with a series of three demographic questions answered using predefined categories-their association with $\mathrm{PD}$, the age of the individual with the disease and their duration of disease.

One central question was asked. This was: What particular aspects of your Parkinson's would, if improved, make the biggest difference to your life? This single question aimed to capture respondents' views on the aspects of PD that have primary, secondary and tertiary importance to quality of life. These aspects could be movement or non-movement symptoms, or side effects related to their PD treatment. Respondents were asked to list up to three aspects in three free text boxes provided with the most important first. No additional prompts were presented in the three text fields for this question. An additional box was presented at the end of the survey for any other comments.

\section{Steering group}

The project's steering group consisted of 3 staff representatives from Parkinson's UK and 6 Patient and Public Involvement (PPI) contributors from the 
charity's Research Support Network. PPI contributors all had direct experience of PD and included 5 people with the condition and 1 partner. To recruit the PPI contributors, an email and role description were sent to all members of the Parkinson's UK network of PPI contributors. The role description outlined key responsibilities, time commitment, timelines for the project and what support was available. PPI contributors expressed their interest in the role by outlining their experience of PD, their interest in the role and any relevant experience of using Excel or analysing data. 10 PPI contributors came forward for the role; and 6 were selected based on their experience and to ensure as much diversity in terms of gender, age and ethnicity as possible. The role of the group was to assist in the interpretation and categorisation of the free-text survey responses, and to provide input on the analysis and presentation of the findings. The steering group met once a month from November 2018 - February 2019 over video-conference.

\section{Data analysis}

Duplicate responses were identified and were removed based on their IP address. The issues reported were recorded as one of 41 specific symptoms or issues related to PD, with some deemed out of scope or uncategorizable (see Supplementary Table 1). This interpretation and sorting exercise was reviewed and finalised by the PPI contributors.

Directed by the PPI contributors, the 41 symptoms or issues were then combined where possible into 24 symptom categories. For instance, responses that mentioned fatigue, tiredness, lack of energy or fitness were grouped together in a broader symptom group called 'Fatigue and energy'. These groups were then organised under 3 main areas: Motor Symptoms, Non-Motor Symptoms and Medication Problems (see Table 2).

Although survey respondents were instructed to list 3 symptoms or aspects of PD in the three free-text boxes in order of importance, some reported more than one symptom in each box while others only reported one or two symptoms in total. It was agreed by the Steering Group that all symptoms reported should be treated as important priorities for patients. Therefore, all symptoms were recorded and given equal weight in the main analysis irrespective of whether they exceeded the three items requested or were reported in the primary, secondary or tertiary response fields.
Pre analysis was performed to compare the types of responses from those with PD $(n=678)$ and those identifying as a carer/partner/family member or friend $(n=104)$, which showed a high degree of similarity suggesting a clear understanding of how the condition affects the individual with PD. Therefore, responses from the carer/partner/family member or friend group were included alongside responses from people with PD within the main analysis.

The responses were subsequently analysed by the duration the individual concerned had been diagnosed with PD. Due to the lower number of respondents in these groups, responses from those that identified as having been diagnosed 11-20 years ago $(n=98)$ or more than 20 years ago $(n=28)$ were combined.

\section{Statistical analysis}

To determine whether statistically significant associations existed between each reported symptom and disease duration, Kruskal-Wallis one-way Analysis of Variance (ANOVA) was applied to each symptom dataset. Post-hoc Dunn's multiple comparisons tests were also applied to determine significance between disease duration groups. Differences with $p<0.05$ were considered statistically significant. Symptoms which demonstrated a significant negative or positive association with disease duration are included in Figs. 3 and 4, respectively. ANOVA p values are described in the text and the results of Dunn's multiple comparisons tests are included in figure legends. Statistical analysis was performed using GraphPad Prism (version 8.4.3 for Windows).

\section{RESULTS}

\section{Survey responses}

A total of 790 responses were included in this study after removal of duplicate submissions. Sample characteristics are presented in Table 1. Some respondents provided more than one distinct symptom or side effect within each of the three boxes provided, while others only reported one or two. Hence the 790 participants responses generated 2443 items when categorised and organised by the steering group.

Of the 2,443 items, 2,295 related to specific symptoms or medication problems. Of these, 1,358 items were categorised as motor symptoms of the condition, 859 items as non-motor symptoms and 78 items as issues with medication (Table 2). The most frequently 
Table 1

Sample characteristics of survey respondents

\begin{tabular}{lc}
\hline Age & \\
$30-49$ & 32 \\
$50-59$ & 117 \\
$60-69$ & 300 \\
$70-79$ & 281 \\
Over 80 & 52 \\
Duration & \\
Less than 2 years ago & 134 \\
2-5 years ago & 313 \\
6-10 years ago & 209 \\
11-20 years ago & 98 \\
More than 20 years ago & 28 \\
Connection & \\
I have Parkinson's & 678 \\
I am a carer/partner/family member or \\
friend & 104 \\
I am a bereaved carer/partner/family \\
member or friend
\end{tabular}

mentioned motor symptoms were tremor, balance and falls, movement problems, walking and stiffness. The most frequently mentioned non-motor symptoms were fatigue and energy, psychological health, sleep problems, pain and unpleasant sensations and cognitive function (Table 2).

Of the remaining 148 items, 113 fell outside the intended scope of the survey. These included the need for improved treatments (46) and a cure (24), better care and management (29), and the desire to maintain independence (14). Although these responses did not directly address the primary question, the project steering group felt that it was important that these responses be reported to highlight the urgency of research to address these issues. The remaining 35 items were uncategorizable.

\section{The importance and complexity of non-motor issues}

Overall, 859 (37.4\%) of in-scope items reported in the survey mentioned aspects of the condition that were categorised as non-motor symptoms (Table 2). The most frequently reported non-motor symptoms were fatigue and energy $(n=180)$, psychological health $(n=154)$, sleep problems $(n=133)$,

Table 2

Responses organised by symptoms reported $(n=2295)$. Symptom categories are given in bold with specific symptoms included listed underneath where relevant. The numbers in brackets represent the total number of respondents that mentioned a symptom within this category. This table includes responses from bereaved partners, family members and friends

\begin{tabular}{|c|c|c|}
\hline Motor Symptoms $(1,358)$ & Non-Motor Symptoms (859) & Medication Problems (78) \\
\hline Tremor (238) & $\begin{array}{l}\text { Fatigue and energy (180) } \\
\text { Fatigue, Tiredness, Lack of energy, } \\
\text { Fitness }\end{array}$ & Medication wearing-off (42) \\
\hline Balance and Falls (154) & Psychological health (154) & Side effects (36) \\
\hline Balance, Falls & $\begin{array}{l}\text { Anxiety, Depression, Apathy, Mood, } \\
\text { Psychological impact, Confidence, } \\
\text { Hallucinations \& Psychosis, Impulsive } \\
\text { and compulsive behaviour }\end{array}$ & \\
\hline Movement problems (148) & Sleep problems (133) & \\
\hline Movement, Moving in bed & Sleep, Daytime sleepiness & \\
\hline Walking (142) & $\begin{array}{l}\text { Pain and unpleasant sensations (110) } \\
\text { Pain, Unpleasant sensations, Restless } \\
\text { legs }\end{array}$ & \\
\hline Stiffness (129) & $\begin{array}{l}\text { Cognitive function (110) } \\
\text { Cognitive impairment, Memory, } \\
\text { Dementia }\end{array}$ & \\
\hline Dexterity and Coordination (118) & Bladder and bowel problems (83) & \\
\hline Dexterity, Coordination & Bladder, Bowel, Incontinence & \\
\hline Speech (107) & $\begin{array}{l}\text { Physiological changes (64) } \\
\text { Sense of smell, Sexual, Skin, Nose, } \\
\text { Breathing, Eyes \& Vision, Heart and } \\
\text { blood pressure, Thermoregulation, Saliva } \\
\text { problems }\end{array}$ & \\
\hline Slowness (93) & Eating and swallowing (25) & \\
\hline Freezing (64) & & \\
\hline Dyskinesia (47) & & \\
\hline Weakness (43) & & \\
\hline Dystonia (42) & & \\
\hline Posture (19) & & \\
\hline Facial expression (14) & & \\
\hline
\end{tabular}




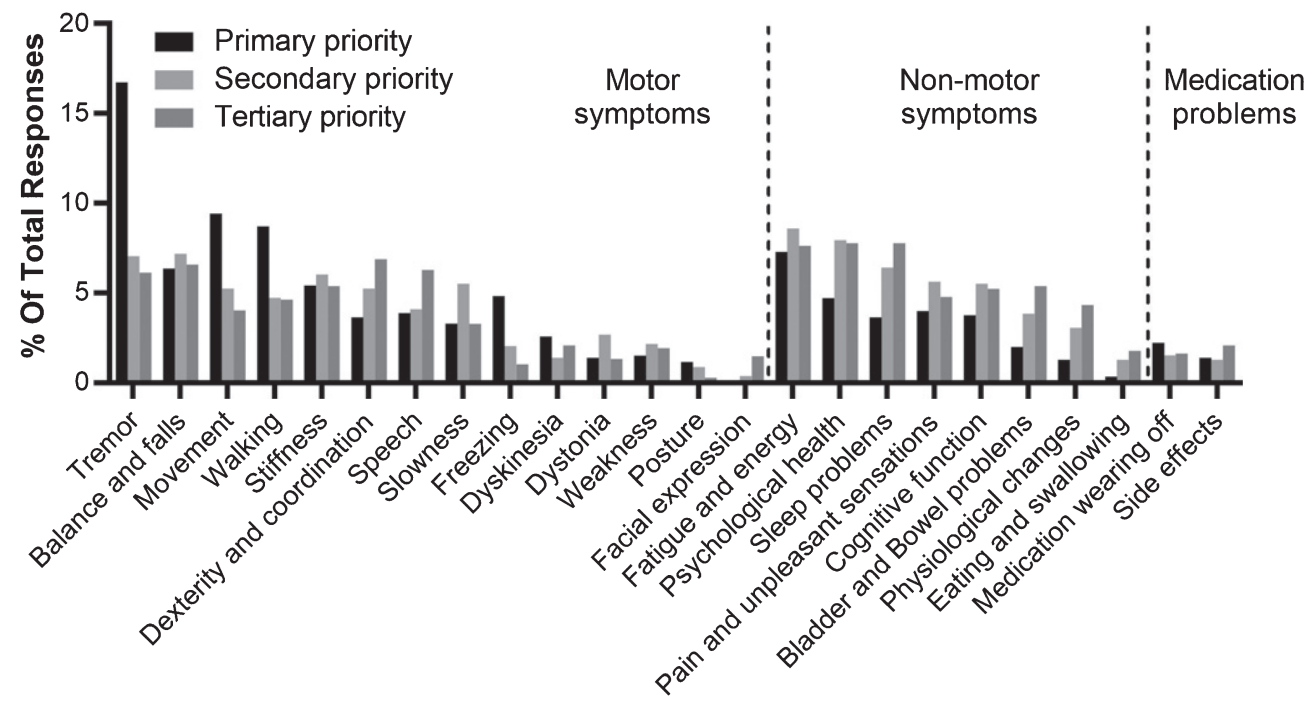

Fig. 1. Symptoms or side effects reported in response to the question "what aspect of Parkinson's do you most wish to see improvement in?" presented by priority. Percentages show the relative frequency of symptoms or side effects reported within primary responses $(n=848)$, secondary responses $(n=779)$, and tertiary responses $(n=668)$.

pain and unpleasant sensations $(n=110)$ and cognitive function $(n=110)$ (Table 2$)$. The complexity and multi-faceted nature of these aspects of the condition is demonstrated by the broad range of terms used to describe them by survey respondents (Supplementary Table 1). This builds upon recent research which found that patient descriptors were more extensive generally for non-motor than motor symptoms [10]. Interestingly, respondents were more likely to report recognisable motor symptoms as their primary priority - in particular tremor, movement or walking problems. In contrast, non-motor symptoms were more often reported as secondary or tertiary issues (Fig. 1).

\section{How priorities change with disease duration}

This study reveals how certain symptoms and medication problems related to PD become more or less important to patients as the condition progresses. The most striking of these is tremor, which is the most frequently reported symptom of PD that respondents wish to improve in this survey. The frequency of mentions of tremor is highest in those living with the condition for 2 years or less (14.4\%) and is reported progressively less frequently, with $4.1 \%$ of those who have lived with PD for 11 years or more mentioning this symptom $(p<0.0001)$ (Figs. 2 and 3). Other symptoms that also become less frequently reported by disease duration include stiffness $(p=0.0026)$ and psychological health $(p=0.0344)$ (Fig. 3).
Issues that appear to become increasingly important as the condition progresses include; problems with walking $(p=0.048)$; balance and falls $(p=0.0035)$; speech problems $(p=0.0009)$; freezing $(p=0.0002)$; dyskinesia $(p<0.0001)$; medication wearing-off ( $p<0.0001)$ (Fig. 4). Indeed, balance and falls rises to become the most important issue for people who have lived with the condition for 11 years or more (Fig. 2).

Non-motor symptoms were highly cited from the very earliest stages of PD. Problems with psychological health, fatigue and energy, cognitive function, and pain and unpleasant sensations all appear in the 10 most frequently reported issues by respondents who had lived with PD for 2 years or less (Fig. 2). Nonmotor symptoms continue to be important throughout the course of PD. Interestingly, only one non-motor symptom, psychological health, showed any association with duration, and difficulties in this domain were reported less frequently with disease duration (Fig. 3).

\section{DISCUSSION}

The results of this survey provide further evidence that the priorities of people affected by PD for improving life are diverse, personal and change substantially with duration of the condition. They reinforce previous research identifying the importance of non-motor issues to quality of life in PD 
a)
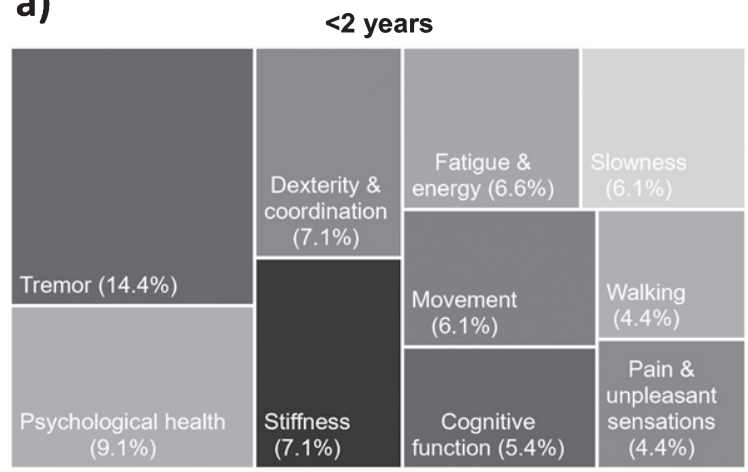

c)

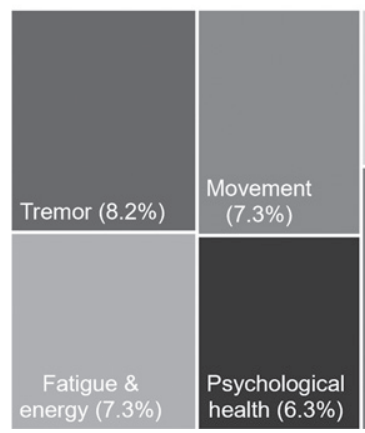

6-10 years

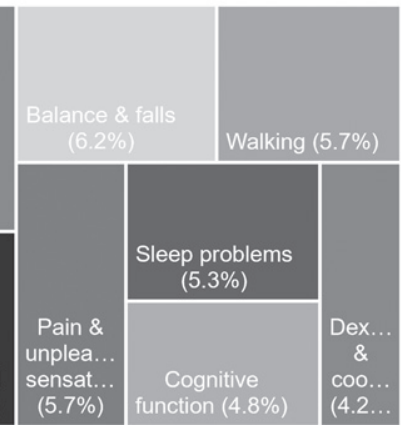

b)

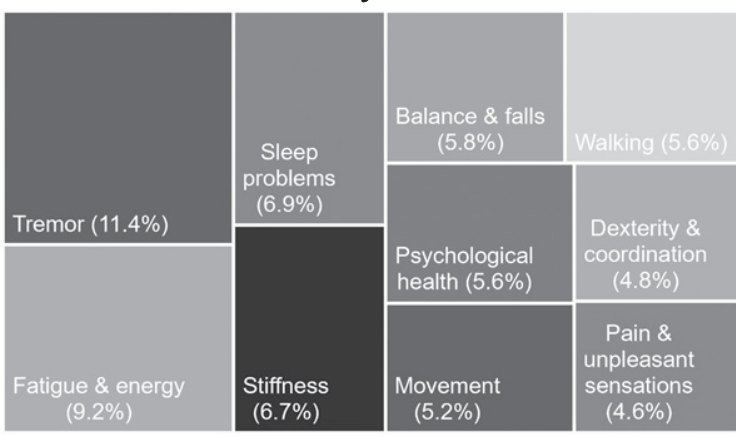

d)

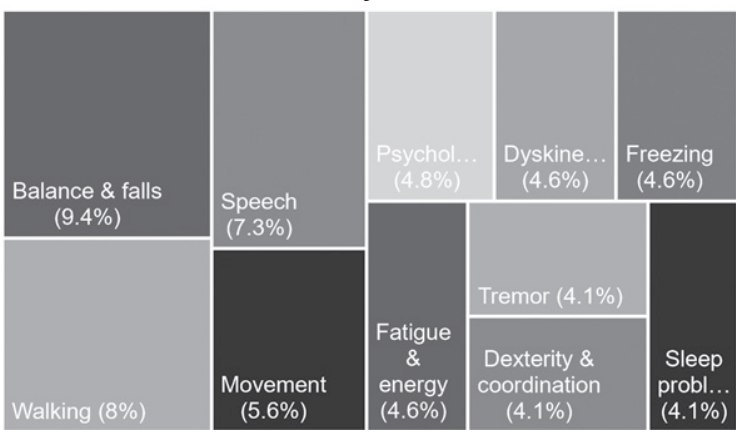

Fig. 2. Top 10 most frequently reported symptoms or side effects respondents wished to see an improvement in by disease duration. The area of the boxes show the relative frequency of the 10 most reported symptoms or side effects respondents diagnosed for (a) $<2$ years $(n=409)$, (b) 2-5 years $(n=648)$, (c) 5-10 years $(n=327)$ and (d) $11+$ years $(n=86)$, wished to see an improvement in, where $\mathrm{n}$ is the total number of categorizable, symptoms or side effects reported. Responses from bereaved partners, family members or friends have been excluded as no duration data is available.

[12-14], and underscore that PD is much more than a movement disorder. However, unlike some previous studies which have suggested that non-motor symptoms become increasingly important to patients over time [15], our findings indicate that non-motor symptoms are important to people affected by PD right from the earliest stages of the condition.

Interestingly, respondents to this survey were likely to mention a motor aspect of the condition as their primary priority, and non-motor symptoms were more frequently reported as secondary or tertiary issues. Our data show that motor symptoms remain important priorities for improving life for people with the condition. Tremor was the most frequently reported aspect of the condition that respondents wished to see an improvement in, in this survey, and is of particular concern to those at an early stage of the disease. This echoes another recently published US study in which tremor was found to be the most bothersome symptom for people with PD [16] alongside research demonstrating that for people living with early stage PD, tremor may cause embarrassment, limit social interactions, and interfere with the ability to perform activities of daily living and simple tasks at home and work [17]. This was also reflected in the free-text comments from respondents in the present survey with many identifying tremor as one of the most noticeable symptoms of the condition and therefore a source of embarrassment and stigma.

This survey indicates that motor symptoms continue to be important for people with PD throughout the course of the condition. Tremor is mentioned progressively less frequently with disease duration (Figs. 2 and 3) while other motor symptoms become more frequently reported with disease duration including balance and falls, walking, freezing, speech and dyskinesia (Fig. 4). Previous research has suggested that tremor progresses more slowly than other motor features [6] and may therefore be overtaken in importance as other issues become more impactful with disease duration.

Where our data adds to existing knowledge is in highlighting the urgent need for better recognition, treatment and management of non-motor symptoms 
a)

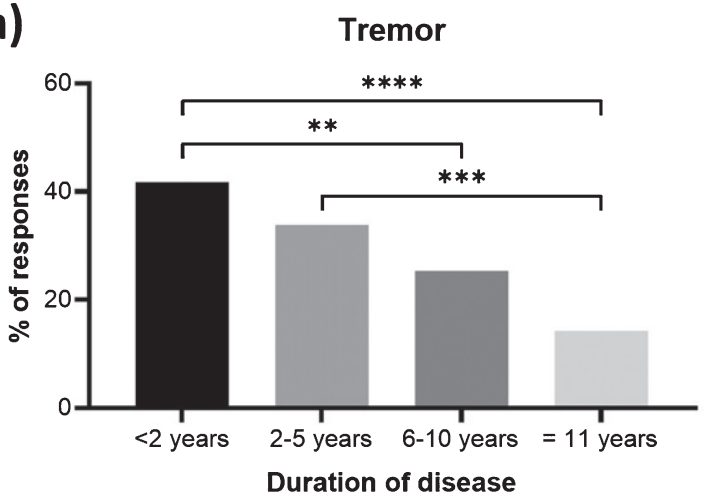

b)

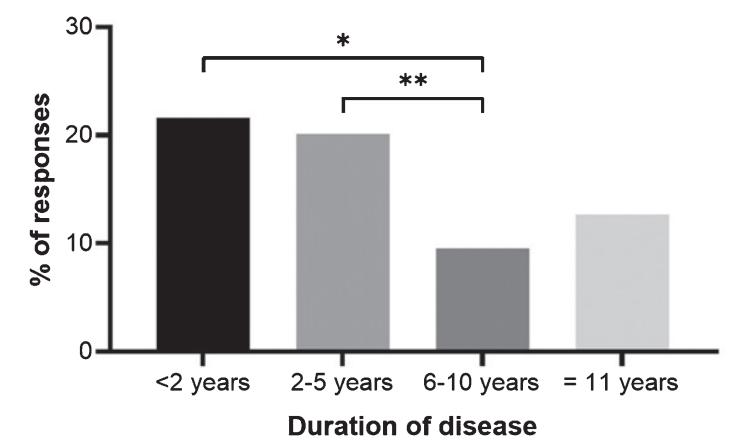

c)

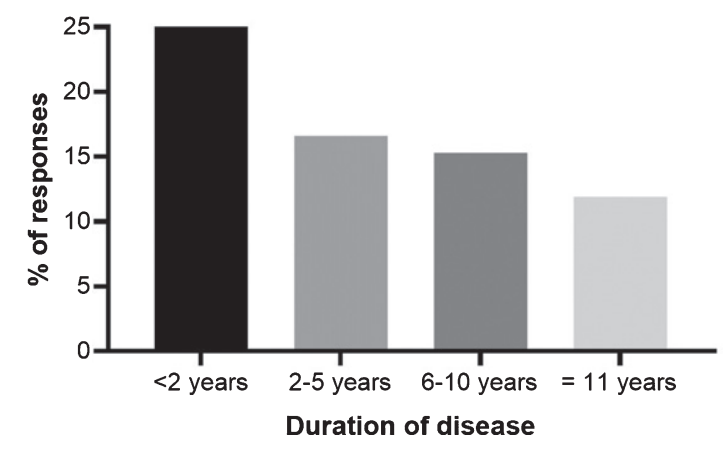

Fig. 3. Symptoms of Parkinson's disease that were reported as a priority for improvement less frequently with disease duration. Percentages show the respondents with a duration of $<2$ years $(n=134), 2-5$ years $(n=313), 6-10$ years $(n=209)$ and $11+$ years $(n=126)$ reporting (a) tremor, (b) stiffness, and (c) psychological health within their 3 priority areas. Statistical significant between duration groups (Dunn's multiple comparisons tests) are presented as asterisks: ${ }^{*} p<0.05 ;{ }^{* *} p<0.01 ;{ }^{* * *} p<0.001 ;{ }^{* * * *} p<0.0001$. Responses from bereaved partners, family members or friends have been excluded as no duration data is available.

for people living with the condition particularly in the very early stages. Previous research has demonstrated that non-motor symptoms have a significant impact on quality of life $[12,18,19]$ and that non-motor symptoms may be present from the very earliest stages, indeed even prodromally [4, 20]. However, the full impact of these issues for patients and their families during these early stages is perhaps less well understood. In this survey, aspects including psychological health, fatigue and energy, cognitive problems, and pain and unpleasant sensations all appear within the top 10 most frequently reported aspects for respondents diagnosed for 2 years or less.

After tremor, issues related to psychological health were the second most frequently reported aspect of the condition that respondents who had been diagnosed with PD for 2 years or less wish to see an improvement in (Fig. 2a). The presence of issues related to psychological health, particularly anxiety and depression, from the very earliest stages of $\mathrm{PD}$ is well established [21]. Our data highlight the impact these issues have on individuals and their families and identifies improved treatment, management and support for this aspect of the condition as an urgent priority at diagnosis.

Issues related to cognitive function, including cognitive impairment, memory problems and dementia, were the eighth most reported issue in those diagnosed for two years or less in this survey. Once again, previous research has demonstrated that changes in cognitive function are relatively common in the early stages of PD [4]. The Parkinson's Progression Markers Initiative's published data suggests that $10 \%$ of people with early, untreated PD may already have cognitive problems [21]. The responses to the present survey reinforce the presence of cognitive changes in the very early stages of the condition and demonstrate the impact and worry these cause people with PD and their families. Interestingly, the results from our survey suggest that cognitive function is more of a concern in those very recently diagnosed than in those who have lived with PD for more than a decade. Respondents diagnosed for two years or less described difficulties with brain fog, mental fuzziness, memory, clarity of thought, and the ability to concentrate and multi-task. These early subtle cognitive changes may impact on many aspects of life as well as causing people to worry about the potential for further deterioration in their cognitive abilities.

Non-motor aspects categorised as 'fatigue and energy' and 'pain and unpleasant sensations' were frequently reported by those recently diagnosed, as well as by those who had lived with PD for longer. A recent review concluded that fatigue is a frequent symptom in PD which appears early and persists as 
a)

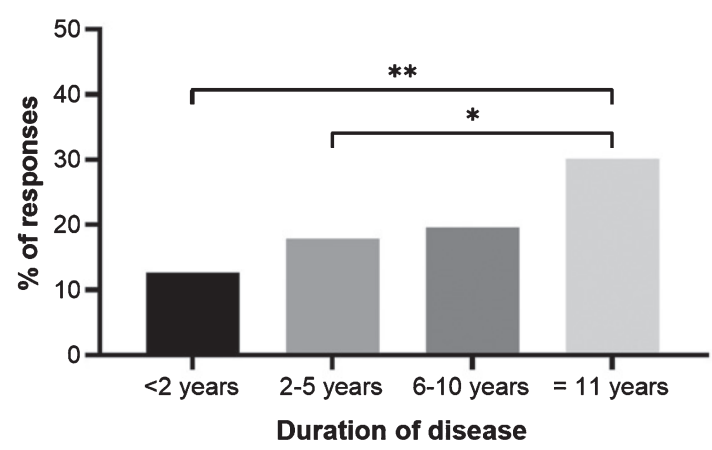

c)

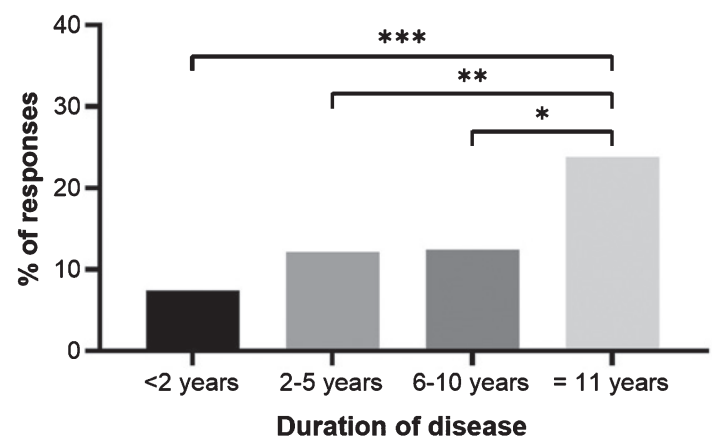

e)

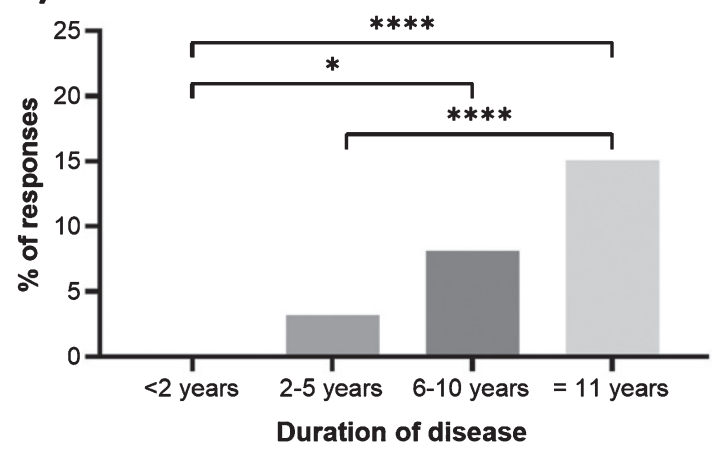

b) Walking

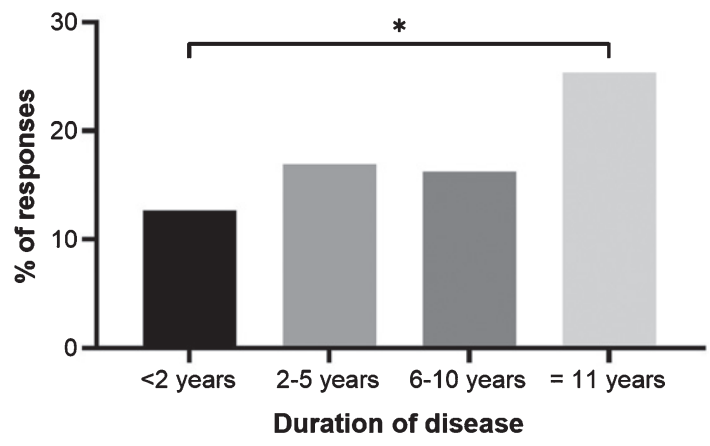

d)

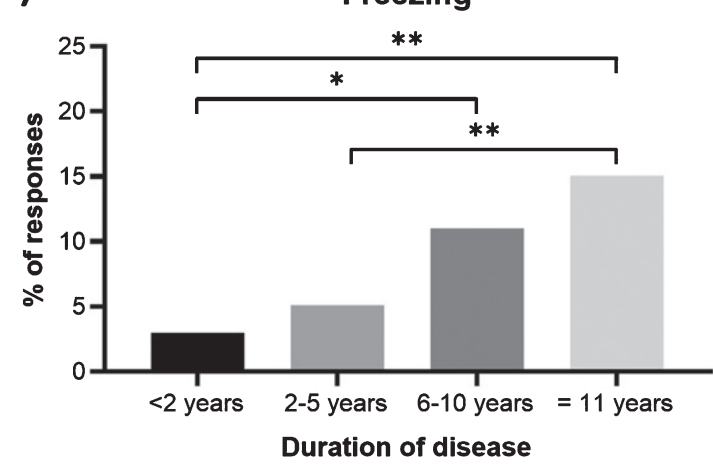

f)

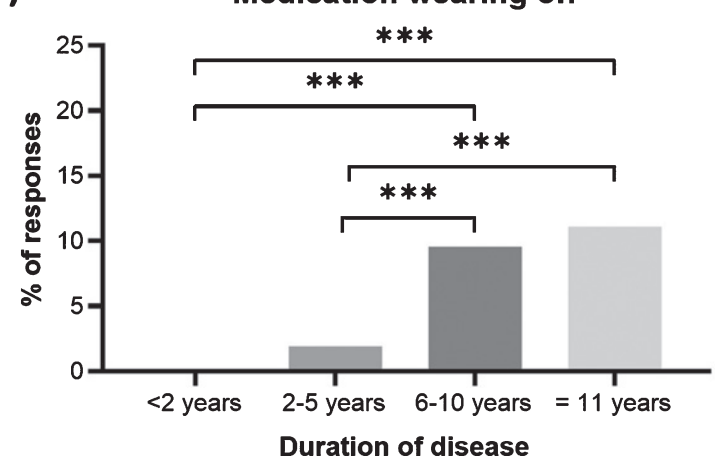

Fig. 4. Symptoms or side effects of Parkinson's disease that were reported as a priority for improvement more frequently with disease duration. Percentages show the respondents with a duration of $<2$ years $(n=134), 2-5$ years $(n=313), 6-10$ years $(n=209)$ and $11+$ years $(n=126)$ reporting (a) balance and falls, (b) walking, (c) speech, (d) freezing, (e) dyskinesia, and (f) medication wearing-off within their 3 priority areas. Statistical significant between duration groups (Dunn's multiple comparisons tests) are presented as asterisks: ${ }^{*} p<0.05$; ${ }^{* *} p<0.01 ;{ }^{* * *} p<0.001 ;{ }^{* * *} p<0.0001$. Responses from bereaved partners, family members or friends have been excluded as no duration data is available.

the condition advances, and called for better recognition including standardised diagnostic criteria for fatigue in PD [22]. Similarly, sleep issues are known to be prevalent in PD and to have a significant impact on quality of life [23]. Our study adds weight to previous calls for greater research efforts to develop better treatments to help manage these debilitating symptoms.

Finally, our data suggest that for people living for 11 years or more with PD, motor aspects of the condition are often the most pressing priorities for improving life. The most frequently reported aspect 
in this group was balance and falls, followed by walking and speech difficulties. These findings corroborate previous studies which have highlighted the increasing burden and complexity of motor symptoms as the condition progresses [4, 6, 7] and emphasise their importance to patients. It is vital that research focuses on developing improved treatments and management strategies that are so important to maintaining independence and quality of life.

The main limitation of this study was the simplicity of the survey. To encourage the maximum possible number of responses the survey was kept deliberately very short. As a result, very limited demographic information was collected regarding age and years since diagnosis, and no data on sex or ethnicity. This study was designed to capture most troublesome symptoms for people with PD with the assumption that they were already receiving appropriate treatment. As a result, there was no information collected regarding medication or other therapies, and we are unable to comment on how treatment may influence symptom priorities. We recommend that future studies collect more detailed demographic information and consider collecting information about medication to enable the relationship between symptoms and medication to be interrogated.

The sample population surveyed, the Parkinson's UK Research Support Network, is a community of individuals who have an active interest in PD research. Separate surveys conducted with this network suggest that they represent a younger, highly educated and predominantly white population whose responses may not be representative of the experiences of the broader PD population in the UK.

A significant challenge in this study was interpreting and appropriately categorizing the free text survey responses, which were wide-ranging. Many responses were ambiguous and required extensive discussion to agree how best to categorise them. This was why it was so important to involve people with PD in this interpretation exercise and is one of the strengths of this study.

One potential confounder of the results of this study is that comorbidities and polypharmacy may account for some of the issues reported in this survey. One recent study using a Scottish primary care database found that only $7.4 \%$ of people with PD had no other recorded conditions [24]. In the present survey, data on comorbidities was not collected but some respondents mentioned that they had difficulties assigning problems to PD rather than to their other conditions.
Overall, this study emphasises the importance of the thorough and ongoing assessment of symptoms throughout the development of the condition as called for by Shin et al. [25]. Each person with PD experiences the condition differently and their own personal priorities for improving life must be at the centre of their care. This also further demonstrates the urgent need for objective measurement tools to accurately capture the most bothersome symptoms for patients to ensure these are properly recognised both in the clinic and in future research [26].

Our data suggest that there should be a greater emphasis upon non-motor issues from the point of diagnosis, especially psychological health, fatigue and energy, pain and unpleasant sensations and cognitive function. Tremor remains an intractable motor symptom that requires improved treatment approaches. This survey identifies problems with balance, walking and falls as the most pressing concerns for patients and their families in the later stages of the condition.

We hope these data will stimulate further research to improve treatments, care and support for people with PD that addresses these important aspects of the condition. We also hope that these research efforts will involve people affected by the condition, their partners and families in developing, designing and conducting these studies to ensure they are truly focused on what matters most to those living with PD.

\section{ACKNOWLEDGMENTS}

We are grateful to the membership of the Parkinson's UK Research Support Network for their participation in this survey and providing their personal perspectives on living with PD. Thanks to the PPI contributors who helped to analyse and categorise the survey responses. Thanks to IH for his help in producing the figures and to Professor David Dexter for editorial assistance and advice.

\section{CONFLICT OF INTEREST}

The authors have no conflict of interest to report.

\section{SUPPLEMENTARY MATERIAL}

The supplementary material is available in the electronic version of this article: https://dx.doi.org/ 10.3233/JPD-202346. 


\section{REFERENCES}

[1] Parkinson's UK (2017) The incidence and prevalence of Parkinson's in the UK, Parkinson's UK website, https:// www.parkinsons.org.uk/news/parkinsons-diagnoses-setincrease-fifth-2025, Posted January 8, 2018, Accessed July 30, 2020.

[2] Moustafa AA, Chakravarthy S, Phillips JR, Gupta A, Keri S, Polner B, Frank MJ, Jahanshahi M (2016) Motor symptoms in Parkinson's disease: A unified framework. Neurosci Biobehav Rev 68, 727-740.

[3] Chaudhuri KR, Healy DG, Schapira AHV (2006) Nonmotor symptoms of Parkinson's disease: Diagnosis and management. Lancet Neurol 5, 235-245.

[4] Kalia LV, Lang AE (2015) Parkinson's disease. Lancet 386, 896-912.

[5] Hoehn MM, Yahr MD (1967) Parkinsonism: Onset, progression, and mortality. Neurology 17, 427-442.

[6] Vu TC, Nutt JG, Holford NH (2012) Progression of motor and nonmotor features of Parkinson's disease and their response to treatment. Br J Clin Pharmacol 74, 267-83.

[7] Alves G, Wentzel-Larsen T, Aarsland D, Larsen JP (2005) Progression of motor impairment and disability in Parkinson disease: A population-based study. Neurology 65, 14361441.

[8] Greenland JC, Williams-Gray CH, Barker RA (2019) The clinical heterogeneity of Parkinson's disease and its therapeutic implications. Eur J Neurosci 49, 328-338.

[9] Lawton M, Ben-Shlomo Y, May MT, Baig F, Barber TR, Klein JC, Swallow DMA, Malek N, Grosset KA, Bajaj N, Barker RA, Williams N, Burn DJ, Foltynie T, Morris HR, Wood NW, Grosset DG, Hu MTM (2018) Developing and validating Parkinson's disease subtypes and their motor and cognitive progression. J Neurol Neurosurg Psychiatry 89, 1279-1287.

[10] Deane KHO, Flaherty H, Daley DJ, Pascoe R, Penhale B, Clarke CE, Sackley C, Storey S (2014) Priority setting partnership to identify the top 10 research priorities for the management of Parkinson's disease. BMJ Open 4, e006434.

[11] Mursaleen LR, Stamford JA, Butterfield T, Edwards G, Kustow P, Kustow PA, Griffith S, Dudgeon GMK, Dudgeon MG (2017) Descriptive symptom terminology used by Parkinson's patients and caregivers. J Parkinsonism Restless Legs Syndr 7, 71-78.

[12] Martinez-Martin P (2011) The importance of non-motor disturbances to quality of life in Parkinson's disease. J Neurol Sci 310, 12-16.

[13] Hinnell C, Hurt CS, Landau S, Brown RG, Samuel M (2011) Non motor versus motor symptoms: How much do they matter to health status in Parkinson's disease? Mov Disord 2, 236-241.

[14] Uebelacker A, Epstein-Lubow G, Lewis T, Broughton MK, Friedman JH (2014) A survey of Parkinson's disease patients: Most bothersome symptoms and coping preferences. J Parkinsons Dis 4, 717-723.
[15] Politis M, Wu K, Molloy S, G Bain P, Chaudhuri KR, Piccini $P$ (2010) Parkinson's disease symptoms: The patient's perspective. Mov Disord 25, 1646-1651.

[16] Hermanowicz N, Castillo-Shell M, McMean A, Fishman J, D’Souza J (2019) Patient and physician perceptions of disease management in Parkinson's disease: Results from a US-based multicenter survey. Neuropsychiatr Dis Treat 15, 1487-1495.

[17] Heusinkveld LE, Hacker ML, Turchan M, Davis TL, Charles D (2018) Impact of tremor on patients with early stage Parkinson's disease. Front Neurol 9, 628.

[18] Barone P, Antonini A, Colosimo C, Marconi R, Morgante L, Avarello TP, Bottacchi E, Cannas A, Ceravolo G, Ceravolo R, Cicarelli G, Gaglio RM, Giglia RM, Iemolo F, Manfredi M, Meco G, Nicoletti A, Pederzoli M, Petrone A, Pisani A, Pontieri FE, Quatrale R, Ramat S, Scala R, Volpe G, Zappulla S, Bentivoglio AR, Stocchi F, Trianni G, Dotto PD; PRIAMO study group (2009) A multicenter assessment of nonmotor symptoms and their impact on quality of life in Parkinson's disease. Mov Disord 24, 1641-1649.

[19] Gallagher DA, Lees AJ, Schrag A (2010) What are the most important non-motor symptoms in patients with Parkinson's disease and are we missing them? Mov Disord 25, 24932500.

[20] Lee HM, Koh SB (2015) Many faces of Parkinson's disease: Non-motor symptoms of Parkinson's disease. Mov Disord 8, 92-97.

[21] Weintraub D, Simuni T, Caspell-Garcia C, Coffey C, Lasch S, Siderowf A, Aarsland D, Barone P, Burn D, Chahine LM, Eberling J, Espay AJ, Foster ED, Leverenz JB, Litvan I, Richard I, Troyer MD, Hawkins KA; Parkinson's Progression Markers Initiative (2015) Cognitive performance and neuropsychiatric symptoms in early, untreated Parkinson's disease. Mov Disord 30, 919-27.

[22] Siciliano M, Trojano L, Santangelo G, De Micco R, Tedeschi G, Tessitore A (2018) Fatigue in Parkinson's disease: A systematic review and meta-analysis. Mov Disord 33, 1712-1723.

[23] Chahine LM, Amara AW, Videnovic A (2017) A systematic review of the literature on disorders of sleep and wakefulness in Parkinson's disease from 2005 to 2015. Sleep Med $\operatorname{Rev~35,~33-50.~}$

[24] McLean G, Hindle JV, Guthrie B, Mercer SW (2017) Co-morbidity and polypharmacy in Parkinson's disease: Insights from a large Scottish primary care database. $B M C$ Neurol 17, 126.

[25] Shin JY, Pohlig RT, Habermann B (2017) Self-reported symptoms of Parkinson's disease by sex and disease duration. West J Nurs Res 39, 1412-1428.

[26] Mathur S, Mursaleen L, Stamford J, DeWitte S, Robledo I, Isaacs T (2017) Challenges of improving patient-centred care in Parkinson's disease. J Parkinsons Dis 7, 163-174. 